\title{
A funcionalidade dos mais idosos ( $\geq 75$ anos): conceitos, perfis e oportunidades de um grupo heterogêneo
}

\author{
The functioning of the oldest old ( $\geq 75$ years): concepts, profiles and opportunities of a \\ heterogeneous group
}

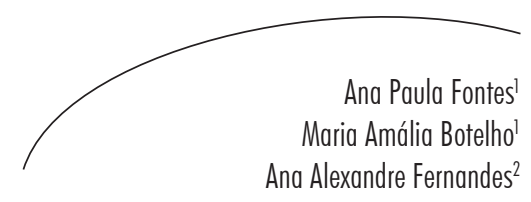

Resumo

Objetivos: Conhecer a funcionalidade de dois grupos de idosos (75-84 anos e $\geq 85$ anos) e sua associação com idade e gênero. Métodos: Trata-se de estudo observacional do tipo analítico e transversal, cuja amostra foi constituída por indivíduos de ambos os sexos com idade $\geq 75$ anos. Foi recolhida informação relativa a condição de saúde e funcionalidade pré-morbilidade. Os instrumentos de coleta de dados foram um questionário de caracterização sociodemográfica e o Método de Avaliação Biopsicossocial. Resultados: A amostra foi constituída por 262 idosos com média de idade de 82,9 \pm 4,86 anos, dos quais $161(61,5 \%)$ eram mulheres. Os mais velhos $(\geq 85$ anos $)$ viviam mais isolados $(p=0,020)$ e tinham menores habilitações literárias $(p=0,027)$, apresentando mais limitações em utilizar escadas $(p=0,015)$, no banho $(p=0,008)$, na continência fecal $(p=0,015)$ e em todas as atividades instrumentais $(p<0,031)$. As mulheres apresentavam maior vulnerabilidade no estado civil ( $\mathrm{p}<0,001)$ e no status econômico ( $\mathrm{p}=0,009)$, enquanto os homens tinham piores resultados nas quedas $(\mathrm{p}=0,003)$ e nos comportamentos de risco $(\mathrm{p}<0,001)$. O desempenho na locomoção e nas atividades básicas e instrumentais era semelhante entre os sexos. Conclusões: As variáveis de natureza social apresentaram-se diferentes quanto à idade e ao gênero. As componentes da funcionalidade - locomoção, atividades diárias básicas e atividades instrumentais - apresentaram maiores limitações nos idosos com idade $\geq 85$ anos, sobretudo as instrumentais. Não se encontraram diferenças na funcionalidade em relação ao gênero.

\section{Abstract}

Objectives: To know the functioning of two groups of seniors (75-84 and $\geq 85$ years) and its association with age and gender. Methodology: This is an observational, analytical and transversal study, with sample composed by elderly individuals with 75 years or

\footnotetext{
Serviço Universitário de Medicina. Faculdade de Ciências Médicas. Universidade Nova de Lisboa. Lisboa, Portugal.

2 Instituto Superior de Ciências Sociais e Políticas. Universidade Técnica de Lisboa. Lisboa, Portugal.

Financiamento: estudo financiado por meio de uma bolsa de investigação para doutoramento, pela Fundação para a Ciência e Tecnologia, no SFRH/BD/60762/2009, concedida à primeira autora do trabalho.
}

Correspondência / Correspondence

Ana Paula Fontes

E-mail: anapaulafontes@gmail.com

Palavras-chave: Idosos. Limitação da Mobilidade. Atividades Cotidianas. Genero e Saúde. Atividades Básicas de Vida Diária. Atividades Instrumentais da Vida Diária. 
older. Data concerned health condition and functioning pre morbidity status. Data collection instruments were a social and demographic characterization questionnaire and the Biopsychosocial Assessment Method. Results: The study sample was composed of 262 elderly, with a mean age of 82,9 \pm 4,86 years, and 161 individuals (61,5\%) were women. The oldest old ( $\geq 85$ years) lived more isolated $(p=0,020)$ and had fewer educational qualifications $(\mathrm{p}=0,027)$, showed greater limitations in climbing stairs $(\mathrm{p}=0,015)$, personal hygiene $(\mathrm{p}=0,008)$, and faecal continence $(\mathrm{p}=0,015)$ and in all instrumental activities $(\mathrm{p}<0,031)$. Women were more vulnerable at marital $(\mathrm{p}<0,001)$ and economic status $(\mathrm{p}=0,009)$, while men had worse results in falls $(\mathrm{p}=0,003)$ and risk factors $(\mathrm{p}<0,001)$. The functional status in mobility and daily activities was similar between the sexes. Conclusions: The oldest old studied had different social state variables vulnerability related to age and gender. However, the difference in functional variables vulnerability was only related to age. The oldest old had more difficulties in some activities, especially those that are instrumental.
Key words: Elderly. Mobility Limitation. Activities of Daily Living. Gender andHealth. Basic Activities of Daily Living. Instrumental Activities of Daily Living.

\section{INTRODUÇÃO}

Talvez um dos mais notáveis desenvolvimentos demográficos dos tempos modernos seja o progressivo envelhecimento populacional, sobretudo o dos grupos mais velhos $(\geq 75$ anos), na língua anglo-saxônica os oldest old. Assistimos, praticamente em todos os países, a um crescimento superior deste grupo em relação aos segmentos mais novos, existindo projeções que referem, para 2050, um aumento quatro vezes superior ao de hoje, ${ }^{1}$ ao qual Portugal não é alheio. Em 2004, estudo publicado pelo Conselho da Europa categorizava-nos, entre os 42 países avaliados, como pertencendo ao grupo dos mais envelhecidos e onde esse fenômeno progrediu de forma exponencial. ${ }^{2}$

Vivemos num processo de explosão sênior que irá progressivamente acentuar-se nas próximas décadas ${ }^{3}$ e que nos obriga a reestruturar de forma global e abrangente suas consequências do ponto de vista econômico, mas sobretudo das políticas sociais e de saúde, pois elas serão, tal como o próprio processo do envelhecimento, gerais, profundas, duradouras e sem precedentes.

Alertados por essas preocupações, vários países têm desenvolvido estudos, alguns longitudinais, no sentido de coletar informação sobre este grupo etário. São exemplos: o Reino
Unido, por meio do General Household Survey, coletou informação durante 20 anos sobre as características demográficas e socioeconômicas dos mais idosos ( $\geq 85$ anos); a Dinamarca desenvolveu três importantes estudos longitudinais que exploram as características dos indivíduos nas últimas etapas de vida: um estudo sobre a população residente nascida em 1905, um sobre os idosos centenários e outro sobre idosos gêmeos com idade $\geq 75$ anos; a Holanda, a partir do Leiden 85-plus Study, tem divulgado diversas informações neste âmbito; a China coletou recentemente dados de 11 mil idosos com idade $\geq 80$ anos; e a Suécia está desenvolvendo uma investigação longitudinal desde 1990.

Em Portugal são inexistentes estudos que abordem especificamente este grupo etário e as informações disponíveis, esgotam-se em aspetos demográficos ou de hábitos de vida, como é o caso do Seneca Study, integrado no Hale Project, o qual não tem em conta a funcionalidade dos indivíduos.

Apesar de os mais idosos apresentarem habitualmente maior número de comorbilidades, associado a índices mais elevados de incapacidade e dependência, mantemos algumas dúvidas sobre a linearidade desse processo. Não existem muitos estudos que avaliem de forma multidimensional a derradeira trajetória funcional deste grupo 
etário. Por outro lado, a progressão linear do declínio funcional associado à idade, tem sido foco de alguns estudos. ${ }^{4,5}$

Conhecer as incapacidades e as potencialidades funcionais deste grupo facilitará o estabelecimento de programas de prevenção adequados, sinalizando fatores de risco emergentes e implementando intervenções eficazes, que resultem na possibilidade de nos anteciparmos às dificuldades e nos forneçam competências para enfrentar esse desafio. Esses procedimentos só serão exequíveis se tiverem por base uma avaliação abrangente, sistematizada e multidimensional da funcionalidade, conforme preconizada pela Organização Mundial da Saúde (OMS) e operacionalizada pela Classificação Internacional de Funcionalidade, Incapacidade e Saúde (CIF).

A CIF é um sistema que descreve de forma multideterminada e multidimensional os aspetos relacionados com a funcionalidade humana. ${ }^{6}$ Seu esquema conceitual se baseia num modelo holístico, biopsicossocial, sobre a saúde e os estados relacionados com a saúde, dividindo-se estruturalmente em duas partes: Funcionalidade/ Incapacidade e Fatores Contextuais.

O termo "funcionalidade" engloba todas as funções do corpo, atividades e participação, indicando os aspetos positivos ou facilitadores, da interação entre um indivíduo com uma condição de saúde e seus fatores contextuais. "Incapacidade" sintetiza as deficiências ou alterações das funções e estruturas, as limitações das atividades e as restrições da participação, ou a magnitude da barreira dos fatores ambientais, revelando assim aspetos negativos da interação entre um indivíduo e seus fatores contextuais ambientais e pessoais. ${ }^{6}$

Os componentes das funções e estruturas, atividades/participação e fatores ambientais estão classificados por meio de categorias, mutuamente exclusivas, que formam as unidades da classificação e estão organizadas numa estrutura hierárquica. Essa hierarquia pode adquirir quatro níveis, que se diferenciam de forma progressiva quanto a sua precisão ou especificidade do conceito, sendo apresentadas por meio de um código alfanumérico. ${ }^{6}$

O envelhecimento não é uma condição de saúde, mas um estado relacionado com a saúde, cuja funcionalidade resulta da interação dinâmica das suas diferentes dimensões, da mesma forma que sua tradução quanto à incapacidade não é considerada um atributo pessoal, mas resultado de uma experiência que engloba algum (ou a totalidade) daqueles fatores.

São objetivos deste estudo: conhecer a funcionalidade de dois grupos de idosos (75-84 anos e $\geq 85$ anos), nomeadamente as caraterísticas de saúde, condição social, da locomoção e das atividades diárias, e identificar a associação entre idade e gênero relativamente ao status funcional das tarefas avaliadas.

\section{MÉTODOS}

Trata-se de estudo observacional do tipo analítico e transversal, de cuja amostra fazem parte os utentes com idade igual ou superior a 75 anos, a viverem anteriormente no domicílio e agora internados nas Unidades de Convalescença e de Média Duração e Reabilitação da Rede Nacional de Cuidados Continuados Integrados da região do Algarve, após terem tido um episódio agudo de internamento hospitalar.

Os dados foram coletados nas primeiras 48 horas de entrada na rede e dizem respeito exclusivamente à condição pré-morbilidade. Coletou-se informação sobre a caraterização sociodemográfica, as diferentes dimensões da funcionalidade - locomoção, autonomia física, autonomia instrumental e condição social. $\mathrm{O}$ estudo foi previamente autorizado pela Comissão de Ética da Administração Regional de Saúde do Algarve, referência 013/10 DEP, de 15/10/2010 e obtido o consentimento informado de todos os participantes, com os dados sido coletados entre abril de 2010 e dezembro de 2011. 
Os instrumentos de coleta de dados foram um questionário de caracterização sociodemográfica, com uma estrutura idêntica à Checklist da $\mathrm{CIF}^{7} \mathrm{e}$ o Método de Avaliação Biopsicossocial (MAB), desenvolvido por Botelho. ${ }^{8}$

O MAB é um "método estruturado, uniformizado, de avaliação tipo rastreio e classificação biopsicossocial de adultos". ${ }^{8}$ Permite caracterizar estados biopsicossociais e funcionais, bem como descrever e detectar perturbações da funcionalidade. Tal como o nome indica, contém três áreas de avaliação, com 12 domínios que aglutinam 19 variáveis, perfazendo na totalidade 56 questões. A pontuação é efetuada numa escala que pode ter até quatro graduações, consoante as características das variáveis, que no seu conjunto permitem atribuir um score aos diferentes domínios, originando por sua vez o perfil funcional do indivíduo. O MAB foi sujeito a um processo de ligação com a CIF, ${ }^{9}$ revelando boa estrutura conceitual e de conceitos significativos, apresentando-se como instrumento abrangente e integrador da avaliação da funcionalidade, conforme preconizado pela OMS.

Caraterizamos a amostra por gênero e dois subgrupos etários: "indivíduos $\leq 84$ anos" e "indivíduos $\geq 85$ anos". Analisamos a associação dessas variáveis com locomoção, autonomia física e autonomia instrumental. As variáveis dos domínios locomoção, autonomia física e autonomia instrumental, que no instrumento são pontuadas em quatro níveis, dicotomizamo-las em "Incapaz/Dependente" (pontuações 0 - incapaz e 1 - dependente de terceiros) e "Autônomo/ Independente" (pontuações 2 - autônomo com meios auxiliares e 3 -independente). As demais variáveis seguiram a cotação original. ${ }^{8}$

A análise dos dados foi feita por meio do programa Statistical Package for Social Sciences $\left(\right.$ SPSS $\left.^{\circledR}\right)$ versão 19. As variáveis categóricas foram descritas por meio de frequências absolutas e relativas; e as numéricas, por meio de medidas de tendência central e de dispersão. A comparação de médias foi efetuada por meio do teste $t$ Student, enquanto para verificar se duas amostras independentes eram ou não iguais em tendência central, se utilizou o teste de Mann-Whitney. Para verificar a independência entre duas variáveis, utilizou-se o teste do Qui-quadrado, e para avaliar seu nível de associação, utilizou-se o Coeficiente de Correlação de Spearman. A análise estatística teve em conta um nível de significância de 0,05.

\section{RESULTADOS}

A amostra foi constituída por 262 idosos cuja média de idade foi de 82,9 \pm 4,86 anos, com valores compreendidos entre os 75 e os 102 anos, dos quais 161 (61,5\%) eram mulheres. As variáveis sociodemográficas e de saúde dicotomizadas pela idade e pelo gênero, encontram-se apresentadas respetivamente nas tabelas 1 e 2 . 
Tabela 1 - Distribuição das caraterísticas sociodemográficas e de saúde por grupo etário e no total. Lisboa, Portugal, 2010-2011.

\begin{tabular}{|c|c|c|c|c|}
\hline Variável & $\begin{array}{c}\text { Idade } \leq 84 \text { anos } \\
\quad(\mathrm{n}=173) \\
\mathrm{n}(\%) ; \text { média } \pm d p\end{array}$ & $\begin{array}{c}\text { Idade } \geq 85 \text { anos } \\
(\mathrm{n}=89) \\
\mathrm{n}(\%) ; \text { média } \pm d p\end{array}$ & $\begin{array}{c}\text { Amostra Total } \\
(\mathrm{n}=262) \\
\mathrm{n}(\%) ; \text { média } \pm d p\end{array}$ & Valor $p$ \\
\hline Idade & $80,1( \pm 2,65)$ & $88,42( \pm 3,14)$ & $82,9( \pm 4,86)$ & \\
\hline Feminino & $80,46( \pm 2,61)$ anos & $88,49( \pm 3,31)$ anos & $82,9( \pm 4,66)$ anos & \\
\hline Masculino & $79,39( \pm 2,62)$ anos & $88,32( \pm 2,97)$ anos & $82,9( \pm 5,18)$ anos & \\
\hline \multicolumn{5}{|l|}{ Sexo } \\
\hline Feminino & $\mathrm{n}=112(64,7)$ & $\mathrm{n}=49(55,1)$ & $\mathrm{n}=161(61,5)$ & 0,128 \\
\hline Masculino & $\mathrm{n}=61(35,3)$ & $\mathrm{n}=40(44,9)$ & $\mathrm{n}=101(38,5)$ & \\
\hline \multicolumn{5}{|c|}{ Quedas sofridas no último ano } \\
\hline Quedas $\geq 4$ & $\mathrm{n}=8(4,6)$ & $\mathrm{n}=10(11,2)$ & $\mathrm{n}=18(6,9)$ & 0,139 \\
\hline Quedas $=2$ a 3 & $\mathrm{n}=31(17,9)$ & $\mathrm{n}=10(11,2)$ & $\mathrm{n}=41(15,6)$ & \\
\hline Quedas $=1$ & $\mathrm{n}=56(32,4)$ & $\mathrm{n}=40(44,9)$ & $\mathrm{n}=96(36,6)$ & \\
\hline Sem quedas & $\mathrm{n}=78(45,1)$ & $\mathrm{n}=29(32,6)$ & $\mathrm{n}=107(40,9)$ & \\
\hline \multicolumn{5}{|l|}{ Status econômico } \\
\hline Sem rendimentos & $\mathrm{n}=10(5,8)$ & $\mathrm{n}=6(6,7)$ & $\mathrm{n}=16(6,1)$ & 0,372 \\
\hline$<250 €$ & $\mathrm{n}=97(56,1)$ & $\mathrm{n}=40(44,9)$ & $\mathrm{n}=137(52,5)$ & \\
\hline$\geq 250 € \mathrm{e}<500 €$ & $\mathrm{n}=39(22,5)$ & $\mathrm{n}=20(22,5)$ & $\mathrm{n}=59(22,5)$ & \\
\hline$\geq 500 €$ & $\mathrm{n}=11(6,4)$ & $\mathrm{n}=9(10,1)$ & $\mathrm{n}=20(7,6)$ & \\
\hline Não sabe/Não responde & $\mathrm{n}=16(9,2)$ & $\mathrm{n}=14(15,7)$ & $\mathrm{n}=30(11,5)$ & \\
\hline \multicolumn{5}{|l|}{ Fumador } \\
\hline $\operatorname{Sim}$ & $\mathrm{n}=22(12,7)$ & $\mathrm{n}=12(13,5)$ & $\mathrm{n}=34(13)$ & 0,770 \\
\hline Não & $\mathrm{n}=151(87,3)$ & $\mathrm{n}=77(86,5)$ & $\mathrm{n}=228(87)$ & \\
\hline \multicolumn{5}{|l|}{ Consumo álcool } \\
\hline Sim & $\mathrm{n}=58(33,5)$ & $\mathrm{n}=29(32,6)$ & $\mathrm{n}=87(33,2)$ & 0,784 \\
\hline Não & $\mathrm{n}=115(66,5)$ & $\mathrm{n}=60(67,4)$ & $\mathrm{n}=175(66,8)$ & \\
\hline \multicolumn{5}{|l|}{ Estado civil } \\
\hline Viúvo/separado/solteiro & $\mathrm{n}=99(57,1)$ & $\mathrm{n}=64(71,9)$ & $\mathrm{n}=163(62,2)$ & 0,020 \\
\hline casado/união de fato & $\mathrm{n}=74(42,9)$ & $\mathrm{n}=25(28,1)$ & $\mathrm{n}=99(37,8)$ & \\
\hline \multicolumn{5}{|l|}{ Escolaridade } \\
\hline 0 ano/analfabeto & $\mathrm{n}=64(37)$ & $\mathrm{n}=45(50,6)$ & $\mathrm{n}=109(41,6)$ & 0,027 \\
\hline 1-6 anos & $\mathrm{n}=93(53,8)$ & $\mathrm{n}=38(42,7)$ & $\mathrm{n}=131(50,0)$ & \\
\hline 7-12 anos & $\mathrm{n}=11(6,4)$ & $\mathrm{n}=4(4,5)$ & $\mathrm{n}=15(5,7)$ & \\
\hline$\geq 13$ anos & $\mathrm{n}=5(2,8)$ & $\mathrm{n}=2(2,2)$ & $\mathrm{n}=7(2,7)$ & \\
\hline \multicolumn{5}{|l|}{ Atividade física semanal } \\
\hline 0 hora & $\mathrm{n}=66(38,2)$ & $\mathrm{n}=47(52,8)$ & $\mathrm{n}=113(43,1)$ & 0,102 \\
\hline$<2$ horas & $\mathrm{n}=17(9,8)$ & $\mathrm{n}=4(4,5)$ & $\mathrm{n}=21(8,0)$ & \\
\hline 2 a 4 horas & $\mathrm{n}=43(24,8)$ & $\mathrm{n}=17(19,1)$ & $\mathrm{n}=60(22,9)$ & \\
\hline$>4$ horas & $\mathrm{n}=47(27,2)$ & $\mathrm{n}=21(23,6)$ & $\mathrm{n}=68(26,0)$ & \\
\hline $\mathrm{N}^{\circ}$ refeiccões habituais/dia & $4,15( \pm 1,06)$ & $4,23( \pm 1,11)$ & $4,18( \pm 1,07)$ & 0,591 \\
\hline
\end{tabular}


Tabela 2 - Distribuição das caraterísticas sociodemográficas e de saúde por gênero. Lisboa, Portugal, 2010-2011.

\begin{tabular}{|c|c|c|c|}
\hline Variável & $\begin{array}{c}\text { Feminino } \\
(\mathrm{n}=161) \\
\mathrm{n}(\%) ; \text { média } \pm d p\end{array}$ & $\begin{array}{c}\text { Masculino } \\
(\mathrm{n}=101) \\
\mathrm{n}(\%) ; \text { média } \pm d p\end{array}$ & Valor $p$ \\
\hline Idade & $82,9( \pm 4,66)$ anos & $82,93( \pm 5,18)$ anos & 0,962 \\
\hline $\begin{array}{l}\text { Quedas sofridas no últim } \\
\text { Quedas } \geq 4 \\
\text { Quedas }=2 \text { a } 3 \\
\text { Quedas }=1 \\
\text { Sem quedas }\end{array}$ & $\begin{array}{c}\mathrm{n}=9(5,6) \\
\mathrm{n}=28(17,4) \\
\mathrm{n}=70(43,5) \\
\mathrm{n}=54(33,5)\end{array}$ & $\begin{array}{c}\mathrm{n}=9(8,9) \\
\mathrm{n}=13(12,9) \\
\mathrm{n}=26(25,7) \\
\mathrm{n}=53(52,5)\end{array}$ & 0,003 \\
\hline $\begin{array}{l}\text { Status econômico } \\
\text { Sem rendimentos } \\
\quad<250 € \\
\geq 250 € \text { e }<500 € \\
\geq 500 € \\
\text { Não sabe/Não responde }\end{array}$ & $\begin{array}{c}\mathrm{n}=15(9,3) \\
\mathrm{n}=85(52,8) \\
\mathrm{n}=33(20,5) \\
\mathrm{n}=9(5,6) \\
\mathrm{n}=19(11,8)\end{array}$ & $\begin{array}{l}\mathrm{n}=1(1,0) \\
\mathrm{n}=52(51,5) \\
\mathrm{n}=26(25,7) \\
\mathrm{n}=11(10,9) \\
\mathrm{n}=11(10,9)\end{array}$ & 0,009 \\
\hline $\begin{array}{l}\text { Fumante } \\
\text { Sim } \\
\text { Não }\end{array}$ & $\begin{array}{c}\mathrm{n}=3(1,9) \\
\mathrm{n}=158(98,1)\end{array}$ & $\begin{array}{l}\mathrm{n}=31(30,7) \\
\mathrm{n}=70(69,3)\end{array}$ & 0,000 \\
\hline $\begin{array}{l}\text { Consumo álcool } \\
\text { Sim } \\
\text { Não }\end{array}$ & $\begin{array}{c}\mathrm{n}=22(13,7) \\
\mathrm{n}=139(86,3)\end{array}$ & $\begin{array}{l}\mathrm{n}=65(64,4) \\
\mathrm{n}=36(35,6)\end{array}$ & 0,000 \\
\hline $\begin{array}{l}\text { Estado civil } \\
\text { Viúvo/separado/solteiro } \\
\text { casado/união de fato }\end{array}$ & $\begin{array}{c}\mathrm{n}=116(72,0) \\
\mathrm{n}=45(28,0)\end{array}$ & $\begin{array}{l}\mathrm{n}=47(46,5) \\
\mathrm{n}=54(53,5)\end{array}$ & 0,000 \\
\hline $\begin{array}{l}\text { Escolaridade } \\
0 \text { Ano/analfabeto } \\
1-6 \text { anos } \\
7-12 \text { anos } \\
\geq 13 \text { anos }\end{array}$ & $\begin{array}{c}\mathrm{n}=71(44,1) \\
\mathrm{n}=81(50,3) \\
\mathrm{n}=6(3,7) \\
\mathrm{n}=3(1,9)\end{array}$ & $\begin{array}{c}\mathrm{n}=38(37,6) \\
\mathrm{n}=50(49,5) \\
\mathrm{n}=8(7,9) \\
\mathrm{n}=5(5,0)\end{array}$ & 0,156 \\
\hline $\begin{array}{l}\text { Atividade física semanal } \\
0 \text { hora } \\
<2 \text { horas } \\
2 \text { a } 4 \text { horas } \\
>4 \text { horas }\end{array}$ & $\begin{array}{c}\mathrm{n}=70(43,5) \\
\mathrm{n}=13(8,1) \\
\mathrm{n}=37(23,0) \\
\mathrm{n}=41(25,4)\end{array}$ & $\begin{array}{c}\mathrm{n}=42(41,6) \\
\mathrm{n}=8(7,9) \\
\mathrm{n}=23(22,8) \\
\mathrm{n}=28(27,7)\end{array}$ & 0,794 \\
\hline $\mathrm{N}^{\circ}$ refeições habituais/dia & $4,29( \pm 1,06)$ & $3,99( \pm 1,07)$ & 0,027 \\
\hline
\end{tabular}


Quando analisamos a amostra relativamente aos grupos etários, verificamos que os idosos $\geq 85$ anos viviam mais sós $(\mathrm{p}=0,020)$ e apresentavam níveis de escolaridade mais baixos ( $\mathrm{p}=0,027)$, mas só o estado civil se mostrou dependente da idade $\left(\mathrm{X}^{2}=0,020\right)$. Quanto ao gênero, as mulheres referiram ter uma condição social mais isolada $(\mathrm{p}<0,001)$, possuírem rendimentos econômicos inferiores $(p=0,001)$ e consumirem mais refeições por dia $(p=0,027)$. Os homens referiram ter caído mais $(\mathrm{p}=0,003)$ e destacaram-se quanto aos hábitos de risco tabágicos e alcoólicos $(\mathrm{p}<0,001)$. A análise estatística inferencial pelo teste do Qui- quadrado permitiu também afirmar dependência entre estas variáveis e o gênero $(\mathrm{p}<0,006)$.

A tabela 3 apresenta os dados e as diferenças encontradas na locomoção, autonomia física e instrumental no que diz respeito à idade. $\mathrm{Na}$ locomoção, os mais idosos apresentavam maior limitação na atividade de subir/descer escadas $(\mathrm{p}=0,015)$, enquanto na autonomia física as maiores dificuldades aconteciam no banho $(\mathrm{p}=0,008)$ e na continência fecal $(\mathrm{p}=0,015)$. Todas as oito atividades instrumentais eram mais desfavoráveis no grupo dos mais velhos.

Tabela 3 - Distribuição das categorias dos domínios da locomoção, autonomia física e autonomia instrumental por grupo etário e no total. Lisboa, Portugal, 2010-2011

\begin{tabular}{|c|c|c|c|c|}
\hline Domínio/Categoria & $\begin{array}{c}\text { Idade } \leq 84 \text { anos } \\
(\mathrm{n}=173) \\
\%\end{array}$ & $\begin{array}{c}\text { Idade } \geq 85 \text { anos } \\
(\mathrm{n}=89) \\
\%\end{array}$ & $\begin{array}{c}\text { Amostra Total } \\
(\mathrm{n}=262) \\
\%\end{array}$ & Valor $p$ \\
\hline \multicolumn{5}{|l|}{ Locomoção } \\
\hline $\begin{array}{l}\text { Andar em casa } \\
\text { Incapaz/Dependente } \\
\text { Autônomo/Independente }\end{array}$ & $\begin{array}{c}n=26(15,0) \\
n=147(85,0)\end{array}$ & $\begin{array}{l}\mathrm{n}=10(11,2) \\
\mathrm{n}=79(88,8)\end{array}$ & $\begin{array}{c}\mathrm{n}=36(13,7) \\
\mathrm{n}=226(86,3)\end{array}$ & 0,390 \\
\hline $\begin{array}{l}\text { Andar na rua } \\
\text { Incapaz/Dependente } \\
\text { Autônomo/Independente }\end{array}$ & $\begin{array}{c}\mathrm{n}=35(20,2) \\
\mathrm{n}=138(79,8)\end{array}$ & $\begin{array}{l}\mathrm{n}=22(24,7) \\
\mathrm{n}=67(75,3)\end{array}$ & $\begin{array}{c}\mathrm{n}=57(21,8) \\
\mathrm{n}=205(78,2)\end{array}$ & 0,419 \\
\hline $\begin{array}{l}\text { Andar em escadas } \\
\text { Incapaz/Dependente } \\
\text { Autônomo/Independente }\end{array}$ & $\begin{array}{c}\mathrm{n}=41(23,7) \\
\mathrm{n}=132(76,3)\end{array}$ & $\begin{array}{l}\mathrm{n}=34(38,2) \\
\mathrm{n}=55(61,8)\end{array}$ & $\begin{array}{l}\mathrm{n}=75(28,6) \\
\mathrm{n}=187(71,4)\end{array}$ & 0,015 \\
\hline Autonomia Física & & & & \\
\hline $\begin{array}{l}\text { Lavar-se/Tomar banho } \\
\text { Incapaz/Dependente } \\
\text { Autônomo/Independente }\end{array}$ & $\begin{array}{l}\mathrm{n}=49(28,3) \\
\mathrm{n}=124(71,7)\end{array}$ & $\begin{array}{l}\mathrm{n}=40(44,9) \\
\mathrm{n}=49(55,1)\end{array}$ & $\begin{array}{l}\mathrm{n}=89(34,0) \\
\mathrm{n}=173(66,0)\end{array}$ & 0,008 \\
\hline $\begin{array}{l}\text { Vestir-se/Despir-se } \\
\text { Incapaz/Dependente } \\
\text { Autônomo/Independente }\end{array}$ & $\begin{array}{c}\mathrm{n}=40(23,1) \\
\mathrm{n}=133(76,9)\end{array}$ & $\begin{array}{l}\mathrm{n}=25(28,1) \\
\mathrm{n}=64(71,9)\end{array}$ & $\begin{array}{l}\mathrm{n}=65(24,8) \\
\mathrm{n}=197(75,2)\end{array}$ & 0,393 \\
\hline $\begin{array}{l}\text { Usar sanit./Bacia/Urinol } \\
\text { Incapaz/Dependente } \\
\text { Autônomo/Independente }\end{array}$ & $\begin{array}{l}\mathrm{n}=35(20,2) \\
\mathrm{n}=138(79,8)\end{array}$ & $\begin{array}{l}\mathrm{n}=23(25,8) \\
\mathrm{n}=66(74,2)\end{array}$ & $\begin{array}{c}\mathrm{n}=58(22,1) \\
\mathrm{n}=204(77,9)\end{array}$ & 0,312 \\
\hline $\begin{array}{l}\text { Deitar/Levantar cama } \\
\text { Incapaz/Dependente } \\
\text { Autônomo/Independente }\end{array}$ & $\begin{array}{c}\mathrm{n}=34(19,7) \\
\mathrm{n}=139(80,3)\end{array}$ & $\begin{array}{l}\mathrm{n}=20(22,5) \\
\mathrm{n}=69(77,5)\end{array}$ & $\begin{array}{c}\mathrm{n}=54(20,6) \\
\mathrm{n}=208(79,4)\end{array}$ & 0,610 \\
\hline
\end{tabular}




\begin{tabular}{|c|c|c|c|c|}
\hline Domínio/Categoria & $\begin{array}{c}\text { Idade } \leq 84 \text { anos } \\
(\mathrm{n}=173) \\
\%\end{array}$ & $\begin{array}{c}\text { Idade } \geq 85 \text { anos } \\
\qquad \begin{array}{c}n=89) \\
\%\end{array}\end{array}$ & $\begin{array}{l}\text { Amostra Total } \\
\qquad \begin{array}{c}(\mathrm{n}=262) \\
\%\end{array}\end{array}$ & Valor $p$ \\
\hline $\begin{array}{l}\text { Sentar/Levantar cadeira } \\
\text { Incapaz/Dependente } \\
\text { Autônomo/Independente }\end{array}$ & $\begin{array}{l}\mathrm{n}=32(18,5) \\
\mathrm{n}=141(81,5)\end{array}$ & $\begin{array}{l}n=16(18,0) \\
n=73(82,0)\end{array}$ & $\begin{array}{c}\mathrm{n}=48(18,3) \\
\mathrm{n}=214(81,7)\end{array}$ & 0,902 \\
\hline $\begin{array}{l}\text { Controlar urina } \\
\text { Incapaz/Dependente } \\
\text { Autônomo/Independente }\end{array}$ & $\begin{array}{c}\mathrm{n}=13(7,5) \\
\mathrm{n}=160(92,5)\end{array}$ & $\begin{array}{l}n=13(14,6) \\
n=76(85,4)\end{array}$ & $\begin{array}{c}\mathrm{n}=26(9,9) \\
\mathrm{n}=236(90,1)\end{array}$ & 0,072 \\
\hline $\begin{array}{l}\text { Controlar fezes } \\
\text { Incapaz/Dependente } \\
\text { Autônomo/Independente }\end{array}$ & $\begin{array}{c}\mathrm{n}=11(6,4) \\
\mathrm{n}=162(93,6)\end{array}$ & $\begin{array}{l}n=14(15,7) \\
n=75(74,3)\end{array}$ & $\begin{array}{c}\mathrm{n}=25(9,5) \\
\mathrm{n}=237(90,5)\end{array}$ & 0,015 \\
\hline $\begin{array}{l}\text { Alimentar-se/Comer } \\
\text { Incapaz/Dependente } \\
\text { Autônomo/Independente }\end{array}$ & $\begin{array}{l}n=18(10,4) \\
n=155(89,6)\end{array}$ & $\begin{array}{l}n=11(12,4) \\
n=78(87,6)\end{array}$ & $\begin{array}{c}n=29(11,1) \\
n=233(88,9)\end{array}$ & 0,645 \\
\hline $\begin{array}{l}\text { Autonomia Instrumental } \\
\text { Usar telefone } \\
\text { Incapaz/Dependente } \\
\text { Autônomo/Independente }\end{array}$ & $\begin{array}{l}\mathrm{n}=49(28,3) \\
\mathrm{n}=124(71,7)\end{array}$ & $\begin{array}{l}n=43(48,3) \\
n=46(51,7)\end{array}$ & $\begin{array}{l}\mathrm{n}=92(35,1) \\
\mathrm{n}=170(74,9)\end{array}$ & 0,002 \\
\hline $\begin{array}{l}\text { Fazer compras } \\
\text { Incapaz/Dependente } \\
\text { Autônomo/Independente }\end{array}$ & $\begin{array}{l}\mathrm{n}=74(42,8) \\
\mathrm{n}=99(57,2)\end{array}$ & $\begin{array}{l}n=54(60,7) \\
n=35(39,3)\end{array}$ & $\begin{array}{l}n=128(48,9) \\
n=134(51,1)\end{array}$ & 0,007 \\
\hline $\begin{array}{l}\text { Preparar refeições } \\
\text { Incapaz/Dependente } \\
\text { Autônomo/Independente }\end{array}$ & $\begin{array}{c}n=68(39,3) \\
n=105(60,7)\end{array}$ & $\begin{array}{l}n=54(60,7) \\
n=35(39,3)\end{array}$ & $\begin{array}{l}n=122(46,6) \\
n=140(53,4)\end{array}$ & 0,001 \\
\hline $\begin{array}{l}\text { Tarefas lida casa } \\
\text { Incapaz/Dependente } \\
\text { Autônomo/Independente }\end{array}$ & $\begin{array}{l}n=79(45,7) \\
n=94(54,3)\end{array}$ & $\begin{array}{l}n=54(60,7) \\
n=35(39,3)\end{array}$ & $\begin{array}{l}n=133(50,8) \\
n=129(49,2)\end{array}$ & 0,024 \\
\hline $\begin{array}{l}\text { Lavar/Tratar Roupa } \\
\text { Incapaz/Dependente } \\
\text { Autônomo/Independente }\end{array}$ & $\begin{array}{c}n=72(41,6) \\
n=101(58,4)\end{array}$ & $\begin{array}{l}n=52(58,4) \\
n=37(41,6)\end{array}$ & $\begin{array}{l}\mathrm{n}=124(47,3) \\
\mathrm{n}=138(52,7)\end{array}$ & 0,011 \\
\hline $\begin{array}{l}\text { Usar transportes } \\
\text { Incapaz/Dependente } \\
\text { Autônomo/Independente }\end{array}$ & $\begin{array}{l}n=79(45,7) \\
n=94(54,3)\end{array}$ & $\begin{array}{l}n=54(60,7) \\
n=35(39,3)\end{array}$ & $\begin{array}{l}n=133(50,8) \\
n=129(49,2)\end{array}$ & 0,024 \\
\hline $\begin{array}{l}\text { Tomar medicamentos } \\
\text { Incapaz/Dependente } \\
\text { Autônomo/Independente }\end{array}$ & $\begin{array}{c}\mathrm{n}=47(27,2) \\
\mathrm{n}=126(72,8)\end{array}$ & $\begin{array}{l}n=36(40,4) \\
n=53(59,6)\end{array}$ & $\begin{array}{c}\mathrm{n}=83(31,7) \\
\mathrm{n}=179(68,3)\end{array}$ & 0,031 \\
\hline $\begin{array}{l}\text { Gerenciar dinheiro } \\
\text { Incapaz/Dependente } \\
\text { Autônomo/Independente }\end{array}$ & $\begin{array}{l}\mathrm{n}=60(34,7) \\
\mathrm{n}=113(65,3)\end{array}$ & $\begin{array}{l}n=45(50,6) \\
n=44(49,4)\end{array}$ & $\begin{array}{l}\mathrm{n}=105(40,1) \\
\mathrm{n}=157(59,9)\end{array}$ & 0,015 \\
\hline
\end{tabular}


Em relação ao sexo, mulheres e homens apresentavam comportamentos semelhantes nas diferentes categorias da locomoção e das atividades/participação (tabela 4).

Tabela 4 - Distribuição das categorias dos domínios da locomoção, autonomia física e autonomia instrumental por gênero. Lisboa, Portugal, 2010-2011.

\begin{tabular}{|c|c|c|c|}
\hline Domínio/Categoria & $\begin{array}{c}\text { Feminino } \\
(\mathrm{n}=161) \\
\%\end{array}$ & $\begin{array}{c}\text { Masculino } \\
(\mathrm{n}=101) \\
\%\end{array}$ & Valor $p$ \\
\hline \multicolumn{4}{|l|}{ Locomoção } \\
\hline $\begin{array}{l}\text { Andar em casa } \\
\text { Incapaz/Dependente } \\
\text { Autônomo/Independente }\end{array}$ & $\begin{array}{c}\mathrm{n}=23(14,3) \\
\mathrm{n}=138(85,7)\end{array}$ & $\begin{array}{l}\mathrm{n}=13(12,9) \\
\mathrm{n}=87(86,1)\end{array}$ & 0,770 \\
\hline $\begin{array}{l}\text { Andar na rua } \\
\text { Incapaz/Dependente } \\
\text { Autônomo/Independente }\end{array}$ & $\begin{array}{c}\mathrm{n}=39(24,2) \\
\mathrm{n}=122(75,8)\end{array}$ & $\begin{array}{c}\mathrm{n}=18(17,8) \\
\mathrm{n}=83(82,2)\end{array}$ & 0,238 \\
\hline $\begin{array}{l}\text { Andar em escadas } \\
\text { Incapaz/Dependente } \\
\text { Autônomo/Independente }\end{array}$ & $\begin{array}{l}\mathrm{n}=49(30,4) \\
\mathrm{n}=112(69,6)\end{array}$ & $\begin{array}{l}\mathrm{n}=26(25,8) \\
\mathrm{n}=75(74,2)\end{array}$ & 0,442 \\
\hline Autonomia Física & & & \\
\hline $\begin{array}{l}\text { Lavar-se/Tomar banho } \\
\text { Incapaz/Dependente } \\
\text { Autônomo/Independente }\end{array}$ & $\begin{array}{c}n=53(32,9) \\
n=108(67,1)\end{array}$ & $\begin{array}{l}\mathrm{n}=36(35,6) \\
\mathrm{n}=65(64,4)\end{array}$ & 0,610 \\
\hline $\begin{array}{l}\text { Vestir-se/Despir-se } \\
\text { Incapaz/Dependente } \\
\text { Autônomo/Independente }\end{array}$ & $\begin{array}{c}\mathrm{n}=40(24,8) \\
\mathrm{n}=121(75,2)\end{array}$ & $\begin{array}{l}\mathrm{n}=25(24,8) \\
\mathrm{n}=76(75,2)\end{array}$ & 0,978 \\
\hline $\begin{array}{l}\text { Usar sanit./Bacia/Urinol } \\
\text { Incapaz/Dependente } \\
\text { Autônomo/Independente }\end{array}$ & $\begin{array}{l}\mathrm{n}=37(23,0) \\
\mathrm{n}=124(77,0)\end{array}$ & $\begin{array}{l}\mathrm{n}=21(20,8) \\
\mathrm{n}=80(79,2)\end{array}$ & 0,709 \\
\hline $\begin{array}{l}\text { Deitar/Levantar cama } \\
\text { Incapaz/Dependente } \\
\text { Autônomo/Independente }\end{array}$ & $\begin{array}{c}\mathrm{n}=31(19,3) \\
\mathrm{n}=130(80,7)\end{array}$ & $\begin{array}{l}\mathrm{n}=23(22,8) \\
\mathrm{n}=78(77,2)\end{array}$ & 0,469 \\
\hline $\begin{array}{l}\text { Sentar/Levantar cadeira } \\
\text { Incapaz/Dependente } \\
\text { Autônomo/Independente }\end{array}$ & $\begin{array}{c}\mathrm{n}=27(16,8) \\
\mathrm{n}=134(83,2)\end{array}$ & $\begin{array}{l}\mathrm{n}=21(20,8) \\
\mathrm{n}=80(79,2)\end{array}$ & 0,392 \\
\hline $\begin{array}{l}\text { Controlar urina } \\
\text { Incapaz/Dependente } \\
\text { Autônomo/Independente }\end{array}$ & $\begin{array}{c}n=15(9,3) \\
n=146(90,7)\end{array}$ & $\begin{array}{l}\mathrm{n}=11(10,9) \\
\mathrm{n}=90(89,1)\end{array}$ & 0,660 \\
\hline $\begin{array}{l}\text { Controlar fezes } \\
\text { Incapaz/Dependente } \\
\text { Autônomo/Independente }\end{array}$ & $\begin{array}{c}n=15(9,3) \\
n=146(90,7)\end{array}$ & $\begin{array}{c}\mathrm{n}=10(9,9) \\
\mathrm{n}=91(90,1)\end{array}$ & 0,856 \\
\hline $\begin{array}{l}\text { Alimentar-se/Comer } \\
\text { Incapaz/Dependente } \\
\text { Autônomo/Independente }\end{array}$ & $\begin{array}{c}n=17(10,6) \\
n=144(89,4)\end{array}$ & $\begin{array}{l}\mathrm{n}=12(11,9) \\
\mathrm{n}=89(88,1)\end{array}$ & 0,719 \\
\hline
\end{tabular}




\begin{tabular}{|c|c|c|c|}
\hline Domínio/Categoria & $\begin{array}{c}\text { Feminino } \\
(\mathrm{n}=161) \\
\%\end{array}$ & $\begin{array}{l}\text { Masculino } \\
(\mathrm{n}=101) \\
\%\end{array}$ & Valor $p$ \\
\hline \multicolumn{4}{|l|}{ Autonomia Instrumental } \\
\hline $\begin{array}{l}\text { Usar telefone } \\
\text { Incapaz/Dependente } \\
\text { Autônomo/Independente }\end{array}$ & $\begin{array}{c}n=51(31,7) \\
n=110(68,3)\end{array}$ & $\begin{array}{l}n=41(40,6) \\
n=60(59,4)\end{array}$ & 0,126 \\
\hline $\begin{array}{l}\text { Fazer compras } \\
\text { Incapaz/Dependente } \\
\text { Autônomo/Independente }\end{array}$ & $\begin{array}{l}n=78(48,4) \\
n=83(51,6)\end{array}$ & $\begin{array}{l}n=50(49,5) \\
n=51(50,5)\end{array}$ & 0,808 \\
\hline $\begin{array}{l}\text { Preparar refeições } \\
\text { Incapaz/Dependente } \\
\text { Autônomo/Independente }\end{array}$ & $\begin{array}{l}n=69(42,9) \\
n=92(57,1)\end{array}$ & $\begin{array}{l}n=53(52,5) \\
n=48(47,5)\end{array}$ & 0,111 \\
\hline $\begin{array}{l}\text { Tarefas da casa } \\
\text { Incapaz/Dependente } \\
\text { Autônomo/Independente }\end{array}$ & $\begin{array}{l}\mathrm{n}=79(49,1) \\
\mathrm{n}=82(50,9)\end{array}$ & $\begin{array}{l}n=55(53,5) \\
n=47(46,5)\end{array}$ & 0,439 \\
\hline $\begin{array}{l}\text { Lavar/Tratar roupa } \\
\text { Incapaz/Dependente } \\
\text { Autônomo/Independente }\end{array}$ & $\begin{array}{l}n=71(44,1) \\
n=90(55,9)\end{array}$ & $\begin{array}{l}n=53(52,5) \\
n=48(47,5)\end{array}$ & 0,162 \\
\hline $\begin{array}{l}\text { Usar transportes } \\
\text { Incapaz/Dependente } \\
\text { Autônomo/Independente }\end{array}$ & $\begin{array}{l}n=82(50,9) \\
n=79(49,1)\end{array}$ & $\begin{array}{l}n=51(50,5) \\
n=50(49,5)\end{array}$ & 0,991 \\
\hline $\begin{array}{l}\text { Tomar medicamentos } \\
\text { Incapaz/Dependente } \\
\text { Autônomo/Independente }\end{array}$ & $\begin{array}{c}\mathrm{n}=47(29,1) \\
\mathrm{n}=114(70,8)\end{array}$ & $\begin{array}{l}n=36(35,6) \\
n=65(64,4)\end{array}$ & 0,252 \\
\hline $\begin{array}{l}\text { Gerenciar dinheiro } \\
\text { Incapaz/Dependente } \\
\text { Autônomo/Independente }\end{array}$ & $\begin{array}{l}\mathrm{n}=62(38,5) \\
\mathrm{n}=99(61,5)\end{array}$ & $\begin{array}{l}\mathrm{n}=43(42,6) \\
\mathrm{n}=58(57,4)\end{array}$ & 0,473 \\
\hline
\end{tabular}

$\mathrm{Na}$ tabela 5, encontram-se os resultados da associação entre idade, gênero e diferentes variáveis estudadas. A independência entre a idade e as categorias dos domínios da locomoção, autonomia física e instrumental, teve comportamento igual às diferenças encontradas entre as duas subamostras. A intensidade e a direção dessa associação se revelaram sempre positivas e fracas. Nas tarefas relacionadas com a locomoção, isso se verificou no subir/descer escadas $\left(r_{s}=0,150\right)$, na autonomia física, no banho $\left(r_{s}=0,165\right)$, na continência fecal $\left(r_{s}=0,150\right)$ e na alimentação $\left(r_{s}=0,029\right)$. Nas atividades instrumentais, observamos associação em todas as categorias, sendo que esta se revelou também sempre positiva e fraca, com variações de $r_{s}=0,134$ (tomar medicamentos) a $\mathrm{r}_{\mathrm{s}}=0,201$ (preparar refeições). Detectamos independência entre o gênero e todas as categorias da locomoção, da autonomia física e da autonomia instrumental. 
Tabela 5 - Associação da idade e do gênero com as diferentes categorias da locomoção, atividades básicas e instrumentais. Lisboa, Portugal, 2010-2011.

\begin{tabular}{|c|c|c|}
\hline & IDADE & GÊNERO \\
\hline \multicolumn{3}{|l|}{ Locomoção } \\
\hline \multirow[t]{2}{*}{ Andar em casa } & $X^{2}=0,389$ & $X^{2}=0,770$ \\
\hline & $\mathrm{p}=0,391$ & $\mathrm{p}=0,771$ \\
\hline \multirow[t]{2}{*}{ Andar na rua } & $\mathrm{X}^{2}=0,418$ & ${ }^{2} \mathrm{X}=0,237$ \\
\hline & $\mathrm{p}=0,420$ & $\mathrm{p}=0,238$ \\
\hline \multirow[t]{3}{*}{ Andar em escadas } & $\mathrm{X}^{2}=0,015$ & ${ }^{2} \mathrm{X}=0,441$ \\
\hline & $\mathrm{p}=0,015$ & $\mathrm{p}=0,443$ \\
\hline & r Spearman $=0,150$ & \\
\hline \multicolumn{3}{|l|}{ Autonomia Física } \\
\hline \multirow[t]{3}{*}{ Lavar-se/Tomar banho } & $\mathrm{X}^{2}=0,008$ & $X^{2}=0,610$ \\
\hline & $\mathrm{p}=0,008$ & $\mathrm{p}=0,611$ \\
\hline & $\mathrm{r}$ Spearman $=0,165^{1}$ & \\
\hline \multirow[t]{2}{*}{ Vestir-se/Despir-se } & $X^{2}=0,392$ & $X^{2}=0,978$ \\
\hline & $\mathrm{p}=0,394$ & $\mathrm{p}=0,978$ \\
\hline \multirow[t]{2}{*}{ Usar Sanit./Bacia/Urinol } & $\mathrm{X}^{2}=0,312$ & $\mathrm{X}^{2}=0,708$ \\
\hline & $\mathrm{p}=0,313$ & $\mathrm{p}=0,709$ \\
\hline \multirow[t]{2}{*}{ Deitar/Levantar Cama } & $X^{2}=0,609$ & $X^{2}=0,468$ \\
\hline & $\mathrm{p}=0,611$ & $\mathrm{p}=0,470$ \\
\hline \multirow[t]{2}{*}{ Sentar/Levantar cadeira } & $\mathrm{X}^{2}=0,901$ & $X^{2}=0,391$ \\
\hline & $\mathrm{p}=0,902$ & $\mathrm{p}=0,393$ \\
\hline \multirow[t]{2}{*}{ Controlar urina } & $\mathrm{X}^{2}=0,071$ & $X^{2}=0,659$ \\
\hline & $\mathrm{p}=0,072$ & $\mathrm{p}=0,660$ \\
\hline \multirow[t]{3}{*}{ Controlar fezes } & $\mathrm{X}^{2}=0,015$ & $\mathrm{X}^{2}=0,855$ \\
\hline & $\mathrm{p}=0,015$ & $\mathrm{p}=0,856$ \\
\hline & r Spearman $=0,150$ & \\
\hline \multirow[t]{2}{*}{ Alimentar-se/Comer } & $X^{2}=0,000$ & $\mathrm{X}^{2}=0,719$ \\
\hline & $\mathrm{p}=0,646$ & $\mathrm{p}=0,720$ \\
\hline
\end{tabular}




\begin{tabular}{|c|c|c|}
\hline \multicolumn{3}{|c|}{ Autonomia Instrumental } \\
\hline \multirow[t]{3}{*}{ Usar telefone } & $X^{2}=0,001$ & $X^{2}=0,125$ \\
\hline & $\mathrm{p}=0,001$ & $\mathrm{p}=0,126$ \\
\hline & r Spearman $=0,197^{1}$ & \\
\hline \multirow[t]{3}{*}{ Fazer compras } & $\mathrm{X}^{2}=0,007$ & $\mathrm{X}^{2}=0,807$ \\
\hline & $p=0,007$ & $\mathrm{p}=0,808$ \\
\hline & r Spearman $=0,167^{1}$ & \\
\hline \multirow[t]{3}{*}{ Preparar refeições } & $\mathrm{X}^{2}=0,001$ & $X^{2}=0,110$ \\
\hline & $\mathrm{p}=0,001$ & $\mathrm{p}=0,111$ \\
\hline & r Spearman $=0,201^{1}$ & \\
\hline \multirow[t]{3}{*}{ Tarefas lida casa } & $\mathrm{X}^{2}=0,024$ & $X^{2}=0,438$ \\
\hline & $\mathrm{p}=0,024$ & $\mathrm{p}=0,440$ \\
\hline & r Spearman $=0,140$ & \\
\hline \multirow[t]{3}{*}{ Lavar/Tratar roupa } & $X^{2}=0,011$ & $\mathrm{X}^{2}=0,162$ \\
\hline & $p=0,011$ & $\mathrm{p}=0,163$ \\
\hline & r Spearman=0,157 & \\
\hline \multirow[t]{3}{*}{ Usar transportes } & $\mathrm{X}^{2}=0,024$ & $\mathrm{X}^{2}=0,991$ \\
\hline & $\mathrm{p}=0,024$ & $\mathrm{p}=0,991$ \\
\hline & r Spearman $=0,140$ & \\
\hline \multirow[t]{3}{*}{ Tomar medicamentos } & $\mathrm{X}^{2}=0,031$ & $\mathrm{X}^{2}=0,251$ \\
\hline & $\mathrm{p}=0,031$ & $\mathrm{p}=0,253$ \\
\hline & r Spearman $=0,134$ & \\
\hline \multirow[t]{3}{*}{ Gerenciar dinheiro } & $X^{2}=0,014$ & $X^{2}=0,472$ \\
\hline & $\mathrm{p}=0,014$ & $\mathrm{p}=0,474$ \\
\hline & r Spearman=0,152 & \\
\hline
\end{tabular}

${ }^{1}$ Nível de significância de 99\%.

\section{DISCUSSÃO}

São vários os estudos que apontam maior longevidade das mulheres, associada, no entanto, a maior incapacidade biológica e pior perceção de saúde. ${ }^{10} \mathrm{Na}$ nossa amostra tal linearidade não se verificou. A percentagem de mulheres, bem como a sua média de idades, não revelou diferença significativa para os homens. A interpretação desses resultados sugere que os homens apresentavam longevidade superior àquela que habitualmente costuma ocorrer. O estado civil e a escolaridade poderão ter contribuído para esta situação, conforme analisaremos posteriormente, pois são indicadores responsáveis pelas diferenças que ocorrem na saúde e também como tendo influência na longevidade e mortalidade dos indivíduos. ${ }^{3}$ Alguns estudos sobre a longevidade de homens octogenários têm revelado comportamento semelhante ao que encontrámos. ${ }^{11}$ 
Diferenças por grupo etário

Em relação às variáveis da condição social, observamos diferenças na existência de companheiro e nas habilitações literárias, apresentando-se o grupo dos mais idosos mais vulnerável nestas situações.

Quanto à conjugalidade, a frequência de idosos sem companheiro foi de $62,2 \%$ na amostra total e de $71,9 \%$ no grupo dos mais velhos. Esta condição tende a progredir com o avançar da idade, devido sobretudo ao estado de viuvez, estando muitas vezes associada a maior dependência e mortalidade. ${ }^{12}$

Sobre a escolaridade, Portugal mantém elevada proporção de portugueses sem escolaridade, ou com fracas competências escolares, especialmente nas gerações mais velhas. ${ }^{3}$ Nossa amostra testemunhou essa tendência, com prevalência de $91,6 \%$ de indivíduos sem escolaridade/baixa escolaridade $(\leq 6$ anos), elevando-se esse valor para $93,3 \%$ no grupo com idade $\geq 85$ anos. Este aspeto deverá constituir um desafio para o desenvolvimento de políticas educacionais das futuras gerações idosas, pois a extensão da escolaridade é um determinante que trará vantagens ao nível da literacia em saúde e, por conseguinte, da saúde das populações. ${ }^{3}$

As investigações sugerem que as idades mais avançadas se associam a maior vulnerabilidade na locomoção e atividades básicas e instrumentais. ${ }^{13,14}$ Nos últimos anos, contudo, têm surgido estudos que apontam para uma estabilização ou mesmo diminuição desse declínio funcional, fruto, entre outros, de maior apoio das tecnologias assistenciais e intervenções clínicas e farmacológicas mais eficazes. ${ }^{15-17}$ Podemos afirmar que nossos resultados apontam nesse sentido.

A incapacidade na locomoção costuma apresentar-se elevada nos grupos etários mais velhos. ${ }^{5,18}$ No entanto, em nosso estudo a prevalência de idosos autônomos/independentes na locomoção em casa e na rua obteve bons resultados (86,3\% e 78,2\%, respectivamente), e só a tarefa de subir/descer escadas se revelou de execução mais difícil para os mais velhos. A dificuldade nesta tarefa pode ocorrer em função das várias deficiências que a compõem, como força, sensibilidade, equilíbrio, coordenação ou visão, mas também a algumas condições crônicas, como a hipertensão arterial, a artrite ou a depressão. Este processo inibe a capacidade e o desempenho de algumas tarefas, sobretudo daquelas que são executadas no exterior e que estiveram de acordo com nossos resultados. A par das estratégias habituais para a melhoria da locomoção, a força dos membros superiores, nomeadamente da preensão das mãos, deverá ser avaliada e se necessário melhorada. Esta função permite identificar os riscos associados à limitação da mobilidade, ${ }^{19}$ na medida em que é fundamental no correto desempenho dos auxiliares de marcha e na utilização de corrimãos, correntemente utilizados pela população idosa. Apesar de não termos verificado diferença no gênero, alguns estudos apontam nesse sentido, apresentando-se as mulheres com maior incapacidade na sua execução, sobretudo quando vivem sós. ${ }^{20}$

Em relação à autonomia física, as maiores dificuldades manifestadas nas tarefas foram as mesmas nos dois grupos etários: vestir/despir e lavar/tomar banho, se bem que só esta última se tenha revelado de mais difícil execução para os mais velhos. De entre as atividades diárias, a higiene pessoal é recorrentemente referida como a que maior limitação produz ${ }^{21}$ e que precede habitualmente outras dificuldades básicas. ${ }^{21}$ Desta forma, as equipes de reabilitação deverão estabelecer processos de sinalização precoce e intervenções sistematizadas, por meio de estratégias compensatórias como as tecnologias assistenciais ou as modificações ambientais.

A incontinência fecal apresentou valores mais elevados no grupo mais idoso. Apesar de menos prevalente que a incontinência urinária e por isso sujeita a menos estudos, a incontinência fecal aparece muitas vezes associada à idade e a algumas condições de saúde, como diarreia crônica ou diabetes, ${ }^{22}$ alterando negativamente e de forma importante a funcionalidade dos indivíduos. 
No que diz respeito à autonomia instrumental, em todas as atividades instrumentais avaliadas, os mais idosos apresentavam-se mais vulneráveis, com valores de incapacidade que variaram de $40,4 \%$ (tomar medicamentos) a 60,7\% (fazer compras, preparar refeições, lida da casa e usar transportes). $\mathrm{O}$ desempenho das atividades incluídas neste domínio exige habilidade mais elaborada não só das funções que lhe estão associadas (força dos membros, mobilidade, equilíbrio, coordenação, funções sensoriais, cognição), mas também uma interação adequada com um ambiente externo, com múltiplas solicitações e exigências e nem sempre familiar. Este conjunto se apresenta habitualmente dependente da idade, promovendo maior incapacidade nos grupos mais velhos. ${ }^{23,24}$

\section{Diferenças de gênero}

Apesar de alguns estudos de avaliação multidimensional apontarem genericamente maior incapacidade às mulheres, ${ }^{3,13,25}$ não encontramos essa diferença na nossa amostra. Quando analisamos as diferenças de gênero em relação à condição social, verificamos que as mulheres se encontravam mais sós, com menos recursos econômicos e consumiam mais refeições por dia, enquanto os homens manifestaram mais quedas no último ano e superioridade nos hábitos de risco.

Quanto à conjugalidade, são vários os estudos que apontam maior isolamento às mulheres, relacionado quer com a sua longevidade, quer com a viuvez. ${ }^{18}$

Recursos econômicos: o status econômico ou a estabilidade financeira estão frequentemente associados à escolaridade e à diferenciação profissional e são considerados determinantes sociais que contribuem para uma maior longevidade. ${ }^{3,26}$ Apesar de não termos encontrado diferenças na escolaridade, nem termos analisado a profissão dos homens e mulheres da nossa amostra, os aspetos culturais que conhecemos do nosso país e sobretudo enraizados na população mais idosa, poderão contribuir para explicar a diferença encontrada nesta variável.

Sobre as quedas, constatamos que as mulheres caem habitualmente mais que os homens. ${ }^{27}$ Essa diferença não tem continuidade nas idades mais avançadas, ${ }^{28}$ conforme aconteceu na nossa amostra. Este resultado deve sinalizar estratégias de modificação ambiental e intervenções direcionadas para as deficiências das funções relacionadas com as quedas.

Um número elevado de refeições por dia é uma das variáveis contempladas nos hábitos e nas dietas alimentares equilibradas, constituindo uma referência na triagem dos grupos de risco. ${ }^{29}$ Este fator, juntamente com a qualidade dos nutrientes, pode melhorar o controle das doenças crônicas, a saúde e a funcionalidade dos mais idosos. ${ }^{30}$ Culturalmente, as mulheres estão mais associadas à aquisição e preparação dos alimentos, o que por si só as predispõe a uma maior tendência para consumir mais refeições.

Sobre comportamentos de risco, o consumo de tabaco nos grupos etários mais idosos é mais prevalente nos homens, observando-se mesmo uma diminuição relativamente às mulheres. ${ }^{18} \mathrm{No}$ nosso país e nas gerações mais velhas, o consumo de álcool aparece ligado substancialmente ao gênero masculino, por questões culturais, de socialização e econômicas, sendo prova disso as doenças e a mortalidade que lhe estão associadas. ${ }^{3}$

A variável sexo é uma das características demográficas com maior peso na diferenciação da autonomia dos idosos, tendendo positivamente para os homens. ${ }^{4,31-33}$ No entanto, e controversamente, encontramos resultados que apontam vantagem funcional básica e instrumental às mulheres. ${ }^{34}$ No nosso estudo, tal como no de Botelho, ${ }^{8}$ não detectamos diferença nas atividades relacionadas ao gênero, podendo este aspecto relacionar-se com as atitudes relativas à saúde demonstradas pelas mulheres. De fato, está amplamente descrito na literatura que alguns comportamentos de risco se relacionam com a funcionalidade, a morbilidade e a mortalidade. ${ }^{31}$ 
As mulheres evidenciam mais comportamentos salutogênicos, sobretudo no que diz respeito aos hábitos alimentares e ao consumo de álcool e tabaco, que poderão explicar nossos resultados.

A independência entre a idade, o gênero e as categorias da locomoção e das atividades básicas e instrumentais esteve de acordo com as diferenças que encontramos entre os grupos, e cuja associação se revelou sempre fraca.

\section{CONCLUSÕES}

Apesar de nosso estudo ser, em termos metodológicos, fundamentalmente descritivo, assinala variáveis consistentes e importantes na definição de estratégias de prevenção e reabilitação dos mais idosos. A ausência da comorbilidade e da polimedicação, como variáveis do estudo, pode ser uma limitação, pois estas são muitas vezes referidas na literatura como associadas ao status funcional deste grupo etário. ${ }^{35}$

Nossos resultados apontam no sentido de que as diferenças entre os mais idosos não se estabelecem de forma linear. Quanto ao perfil social, encontramos os idosos com idade $\geq 85$ anos mais isolados e com menos escolaridade, e as mulheres mais vulneráveis no status econômico e no estado civil, mas pontuando positivamente no número de refeições/dia. Os homens evidenciam-

\section{REFERÊNCIAS}

1. United Nations Population Division. World Population Ageing 1950-2050. Population and Development Review 2002;28(4):814-5.

2. Schoenmaeckers RC. Active ageing in Europe Volume 2: Demographic characteristics of the oldest old. Populations Studies n.47; Council of Europe; 2004.

3. Fernandes AA. Determinantes da mortalidade e da longevidade: Portugal numa perspectiva europeia (UE15, 1991-2001). Anál Social 2007;183:419-43.

4. Aijanseppa S, Notkola IL, Tijhuis M, Van Staveren W, Kromhout D, Nissinen A. Physical functioning in elderly Europeans: 10 year changes in the north and south: the HALE Project. J Epidemiol Community Health 2005;59(5):413-9. se pelos comportamentos de risco apresentando mais quedas e maiores consumos de álcool e tabaco. As diferenças na locomoção e nas atividades ocorreram exclusivamente ligadas à idade, sobretudo as relacionadas com as tarefas instrumentais, cujos resultados foram mais desfavoráveis nos mais idosos.

O desenvolvimento de estratégias metodológicas de avaliação da funcionalidade da população mais idosa tem relevância epidemiológica importante, sobretudo quando consegue caracterizar os aspetos ambientais e pessoais passíveis de melhorar a capacidade e o desempenho das dimensões biológica, psicológica e social dos indivíduos. Por outro lado, os processos de reabilitação devem focar-se na manutenção e melhoria das funções mais ligadas ao processo de senescência que contribuem de forma mais direta para a incapacidade observada nas atividades básicas e instrumentais.

Os aspetos sociais relacionados com a vulnerabilidade funcional deverão ser sinalizados e analisados pelos serviços, sistemas e políticas sociais, no sentido de serem solucionados. São disso exemplo o isolamento social e a baixa escolaridade, que poderão ser minorados por meio da criação de redes informais de apoio comunitário e o desenvolvimento de projetos e ações de formação/educação intergeracional.

5. Pérès K, Helmer C, Letenneur L, JacqminGadda H, Barberger-Gateau P. Ten year change in disability prevalence and related factors in two generations of French elderly community dwellers: data from the PAQUID study. Aging Clin Exp Res 2005;17(3):229-35.

6. World Health Organization. International Classification of Functioning, Disability and Health (ICF). Geneve: WHO; 2001.

7. World Health Organization. ICF Checklist: version 2.1a, Clinician Form for International Classification of Functioning, Disability and Health. 2003 [acesso em 1 mar 2012]. Disponível em: http://www.who.int/ classifications/icf/training/icfchecklist.pdf 
8. Botelho, MA. Autonomia Funcional em Idosos: caracterização multidimensional em idosos utentes de um centro de saúde urbano. Porto: Edições Bial; 2000.

9. Fontes AP, Botelho MA, Fernandes AA. Content comparison of a Biopsychosocial evaluation method and the International Classification of Functioning, Disability and Health (ICF). Educ Gerontol; In press, 2011.(3

10. Case AC, Paxson C. Sex differences in morbidity and mortality. Demography 2005;42(2):189-214.

11. Panagiotakos DB, Chrysohoou C, Siasos G, Zisimos K, Skoumas J, Pitsavos C, et al. Sociodemographic and lifestyle statistics of oldest old people ( $>80$ years) living in ikaria island: the ikaria study. Cardiol Res Pract 2011;24:679187.

12. Manzoli L, Villari P, Pirone G, Boccia A. Marital status and mortality in the elderly: a systematic review and meta-analysis. Soc Sci Med 2007;64(1):77-94.

13. Palacios-Ceña D, Jiménez-García R, HernándezBarrera V, Alonso-Blanco C, Carrasco-Garrido P, Fernández-de-Las-Peñas C. Has the prevalence of disability increased over the past decade (2000-2007) in elderly people? A Spanish population-based survey. J Am Med Dir Assoc 2012;13(2):136-42.

14. Zunzunegui MV, Nunez O, Durban M, García de Yébenes MJ, Otero A. Decreasing prevalence of disability in activities of daily living, functional limitations and poor self-rated health: a 6-year follow-up study in Spain. Aging Clin Exp Res 2006;18(5):352-8.

15. Hung WW, Ross JS, Boockvar KS, Siu AL. Recent trends in chronic disease, impairment and disability among older adults in the United States. BMC Geriatr 2011;18:11:47.

16. Schoeni RF, Freedman VA, Martin LG. Why is latelife disability declining? Milbank Q 2008;86(1):47-89.

17. Freedman VA, Martin LG, Schoeni RF, Cornman JC. Declines in late-life disability: the role of early- and mid-life factors. Soc Sci Med 2008;66(7):1588-602.

18. Tourlouki E, Polychronopoulos E, Zeimbekis A, Tsakountakis N, Bountziouka V, Lioliou E, et al. The 'secrets' of the long livers in Mediterranean islands: the MEDIS study. Eur J Public Health 2010;20(6):659-64.

19. Sallinen J, Stenholm S, Rantanen T, Heliovaara M, Sainio P, Koskinen S. Hand-grip strength cut points to screen older persons at risk for mobility limitation. J Am Geriatr Soc 2010;58(9):1721-6.
20. McLaughlin D, Leung J, Byles J, Dobson A. Living with stairs: functioning in a large cohort of older Australian adults. J Am Geriatr Soc 2011;59(8):1560-2.

21. Naik AD, Concato J, Gill TM. Bathing disability in municipality-living older persons: Common, consequential, and complex. J Am Geriatr Soc 2004;52(11):1805-10

22. Østbye T, Seim A, Krause KM, Feightner J, Hachinski V, Sykes E, et al. A 10-Year Follow-Up of Urinary and Fecal Incontinence among the Oldest Old in the Community: the Canadian Study of Health and Aging. Can J Aging 2004;23(4):319-31.

23. den Ouden ME, Schuurmans MJ, Arts IE, van der Schouw YT. Physical performance characteristics related to disability in older persons: a systematic review. Maturitas 2011;69(3):208-19.

24. Vermeulen J, Neyens JCL, van Rossum E, Spreeuwenberg MD, Witte LP. Predicting ADL disability in community-dwelling elderly people using physical frailty indicators: a systematic review. BMC Geriatr 2011;1:33

25. Virués-Ortega J, Pedro-Cuesta J, Seijo-Martínez M, Saz P, Sánchez-Sánchez F, Rojo-Pérez F, et al. Prevalence of disability in a composite $\geq 75$ year old population in Spain: A screening survey based on the International Classification of Functioning. BMC Public Health 2011;11:176.

26. Stessman J, Hammerman-Rozenberg R, Maaravi Y, Azoulai D, Cohen A. Strategies to enhance longevity and independent function: the Jerusalem Longitudinal Study. Mech Ageing Dev 2005;126(2):327-31.

27. Dhanwal DK, Dennison EM, Harvey NC, Cooper C. Epidemiology of hip fracture: Worldwide geographic variation. Indian J Orthop 2011;45(1):15-22.

28. Lehtola S, Koistinen P, Luukinen H. Falls and injurious falls: late in home-dwelling life. Arch Gerontol Geriatr 2006;42(2):217-24.

29. Schroll M. Aging, food patterns and disability. Forum Nutr 2003;56:256-8.

30. Woo J. Nutritional strategies for successful aging. Med Clin North Am 2011;95(3):477-93.

31. Wister A, Wanless D. A health profile of communityliving nonagenarians in Canada. Can J Aging 2007;26(1):1-18.

32. von Strauss E, Agüero-Torres H, Kareholt I, Winblad $\mathrm{B}$, Fratiglioni L. Women are more disabled in 
basic activities of daily living than men only in very advanced ages: a study on disability, morbidity, and mortality from the Kungsholmen Project. J Clin Epidemiol 2003;56(7):669-77.

33. Nybo H, Gaist D, Jeune B, McGue M, Vaupel JW, Christensen K. Functional status and self-rated health in 2,262 nonagenarians: The Danish 1905 Cohort Survey. J Am Geriatr Soc 2001;49(5):601-9.
34. Pachana NA, McLaughlin D, Leung J, Mckenzie SJ, Dobson A. The effect of having a partner on activities of daily living in men and women aged 8287 years. Maturitas 2011;68(3):286-90.

35. Ravaglia G, Forti P, Lucicesare A, Pisacane N, Rietti E, Patterson C. Development of an easy prognostic score for frailty outcomes in the aged. Age Ageing 2008;37(2):161-6.

Recebido: 26/6/2012

Revisado: $12 / 10 / 2012$

Aprovado: 30/20/2012 